\title{
FRECUENCIA DE Trifolium amabile KUNTH (FABACEAE) EN DOS SITIOS DEL ALTIPLANO DE PUNO, PERÚ
}

\section{FRECUENCY OF Trifolium amabile KUNTH (FABACEAE) AT TWO ECOLOGICAL SITES IN THE HIGH LANDS OF PUNO, PERU}

\author{
Gregorio Argote ${ }^{1}$ Lucrecia Aguirre $^{2}$ y Enrique Flores ${ }^{3}$
}

\begin{abstract}
Resumen
Se estudió la frecuencia de T. amabile y su grado de asociación con otras especies vegetales en dos sitios de pastizal dominados por gramíneas altas, IIpa 3820 msnm y Quimsachata 4300 msnn, así como el grado de correlación entre la presencia de esta especie con variables físico-químicas del suelo. T. amabile estuvo presente en la mayoría de las comunidades vegetales estudiadas pero en porcentajes relativamente bajos, rangos de 1.0 a $8.3 \%$, y asociada positivamente $(\mathrm{p}<0.05)$ con Alchemilla pinnata, Azorella diapensoides, Calamagrostis amoena, Festuca dolichophylla y Muhlenbergia fastigiata, en condiciones de suelos profundos, bien drenados y con un adecuado contenido de fósforo y nitrógeno; lo cual, estaría sugiriendo que estos elementos químicos podrían estar limitando su mayor frecuencia y distribución en el altiplano. Se recomienda realizar estudios para dilucidar el rol de esta leguminosa en el aporte de nitrógeno al suelo y la calidad de la dieta de animales al pastoreo, teniendo en cuenta que el contenido de proteína de la mayoría de las gramíneas nativas, durante gran parte del año se mantiene muy por debajo de niveles recomendables para una buena nutrición del ganado.

Palabras clave: Sitio ecológico, frecuencia, asociaciones vegetales, pastizales, factores abióticos.
\end{abstract}

\begin{abstract}
The frecuency of $T$. amabile, its degree of association with other species and the correlation with soil physical and chemical variables were studied at two Andean grassland sites dominated by tall grasses (Illpa located at $3820 \mathrm{msnm}$ and Quimsachata at $4300 \mathrm{msnm}$ ). Trifolium amabile was present in low percentages, less than $8.3 \%$, in the majority of vegetation communities and its presence was positively associated $(\mathrm{p}<0.05)$ with Alchemilla pinnata, Azorella diapensioides, Calamagrostis amoena, Festuca dolichophylla and Muhlenbergia fastigiata, where deep and well drained soils were present and where phosphorus and nitrogen content where adequate. This suggests that phosphorus and nitrogen could be acting as factors limiting its presence and distribution in the altiplano. It is recommended to conduct studies to clarify the role of this native legume on nitrogen fixation and its potential contribution to diet quality of grazing animals, given most native grasses are low in protein content.
\end{abstract}

Key words: ecological site, frequency, vegetation association, grasslands, abiotic factors.

\section{Introducción.}

Los pajonales constituyen un ecosistema importante en el altiplano puneño, representando más del $70 \%$ de esta región semi árida (Flores, 1991). La composición florística de los pajonales y su patrón de distribución varía dependiendo de la topografía, tipo de suelo y el clima (Tapia \& Flores, 1984; Florez et al., 1992; Cajal et al., 1998; Florez, 2005). La florística de los pajonales está dominada en el estrato alto por gramíneas de los géneros Festuca, Calamagrostis y Stipa, mientras que el estrato bajo lo está por hierbas y gramíneas cortas y en una pequeña proporción por leguminosas (Flores, 1991; Tapia \& Flores, 1984; Wilcox \& Bryant, 1984; Florez, 2005). Algunos estudios señalan que las leguminosas entre ellas Trifolium amabile son poco abundantes y no superan el 5\% de cobertura vegetal (Pasquín et al., 2002). La presencia de leguminosas sin embargo es importante por su capacidad en contribuir a la mejora de la calidad del forraje. Trifolium amabile, es una leguminosa nativa perenne cuya presencia ha sido reportada desde 2000 a más de $4000 \mathrm{msnm}$ (De La Cruz, 2007; Alemán, 1996) y es una de las pocas especies forrajeras de la familia fabaceae que se halla distribuida en las praderas alto andinas de Puno (Chaquilla, 1969). Esta leguminosa es resistente a la sequía, tolerante a bajas temperaturas $\mathrm{y}$, por su excelente valor forrajero (Tapia \& Flores, 1984) podría ser aprovechada en beneficio de una mejor producción ganadera.

Trifolium amabile despertó nuestro interés por su grado de adaptación a condiciones de puna, donde 
muchas leguminosas exóticas tienen pocas posibilidades de prosperar debido a las duras condiciones ambientales, por lo que el presente estudio tuvo como propósito evaluar la influencia del sitio ecológico en la presencia de T. amabile, el grado de asociación con otras especies y características del suelo asociados con su presencia.

\section{Materiales y métodos.}

El estudio se desarrolló a partir de enero 2009 y finalizó en diciembre 2010 en la Región Puno, Perú, en dos zonas, Illpa ubicada entre 3820 a 3900 msnm y Quimsachata ubicada a una altitud de 4000 a 4300 msnm. El estudio abarcó una área de 11000 ha, 3000 correspondientes a Quimsachata y 8000 a Ilpa, alcanzando un total de 20 sitios ecológicos, 10 en cada zona (Figura 1). La zona de Illpa, según el sistema Holdridge se clasifica como Bosque húmedo-Montano Subtropical (Bh - MS) y, se caracteriza por ser de un clima templado-frío, la temperatura media anual oscila de 6.0 a $8.0{ }^{\circ} \mathrm{C}$, con una precipitación anual de 600 a $700 \mathrm{~mm}$. La zona de Quimsachata como Páramo muy húmedo-Subalpino Subtropical (Pmh-SAS), cuya temperatura media anual oscila de 4.5 a $6.0^{\circ} \mathrm{C}$, y una precipitación anual de 500 a $720 \mathrm{~mm}$.

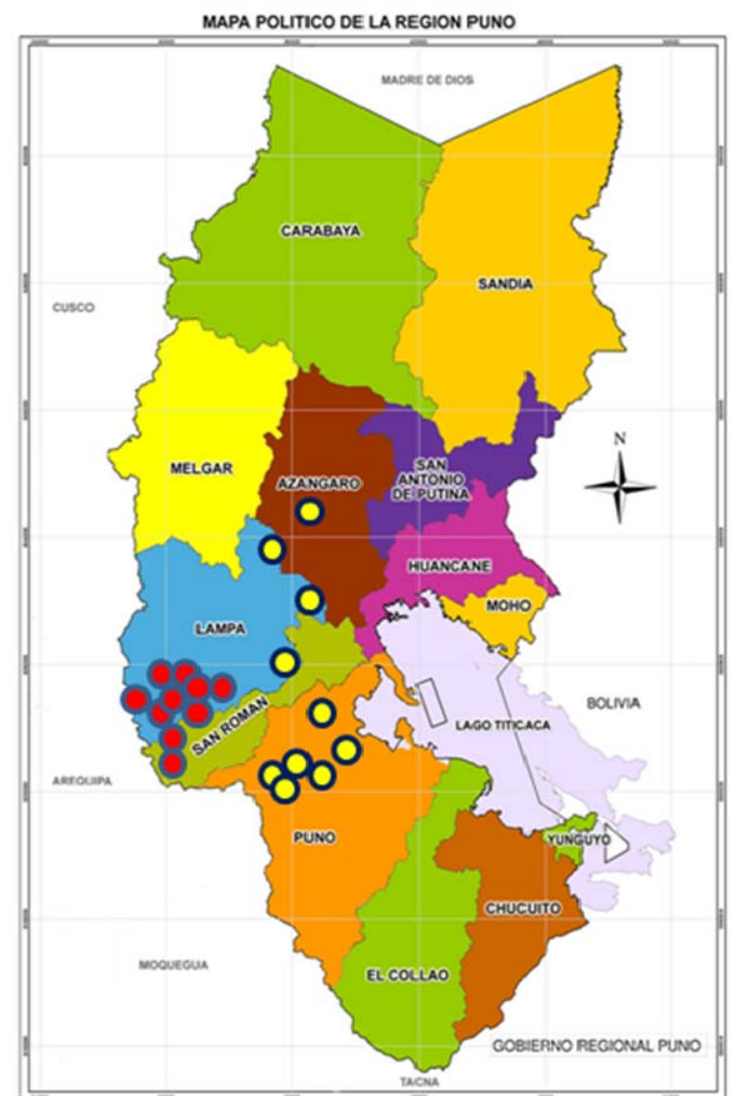

Figura 1. Mapa de localización de las zonas de estudio.
La vegetación presente tanto en la zona de Illpa como en Quimsachata estuvo conformado en un alto porcentaje de gramíneas (> $60 \%$ ) de los géneros Festuca, Calamagrostis, Muhlenbergia, Poa, Aristida, Stipa y Bouteloua, entre otros (Figura 2). Las hierbas ocuparon el segundo grupo de importancia en la estructura de estas comunidades, dentro de éstas especies se encontraron Trifolium amabile y Alchemilla pinnata. Es notorio que en la zona de Quimsachata, exista mayor proporción de arbustos típicos de la zona altiplánica como Parastrephia quadrangularis. Otro grupo de plantas que también está presente son las graminoides como Carex sp y Eleocharis albibracteata.

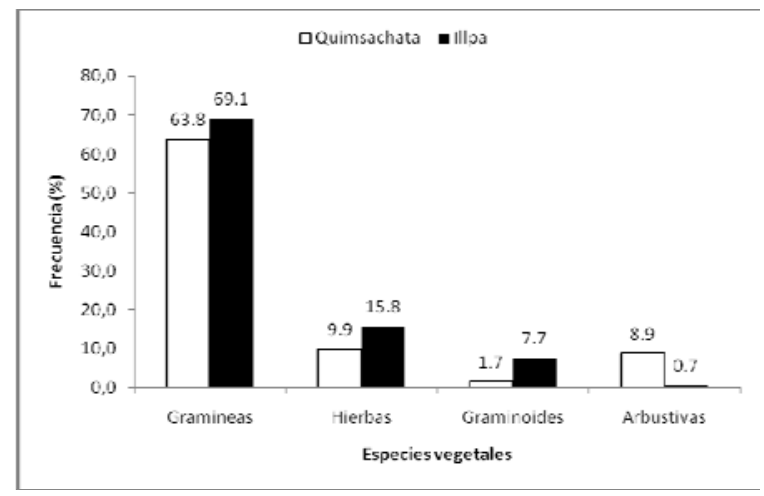

Figura 2. Estructura de las comunidades vegetales en la zona de Illpa y Quimsachata

Los suelos de las áreas estudiadas en la zona de Illpa, corresponden a las series Titicaca, Pupuja, Pucará y Calapuja, que se ubican a lo largo de planicies y cerros, exhiben una textura que varía entre franco arcilloso a franco limoso, pH 5.5 a 5.8; con niveles en nitrógeno bajo, fósforo medio y potasio alto, (ONERN, 1965). En la zona de Quimsachata, los suelos corresponden a las series Huaytapata, Quimsachata y Atun Mocco. Son suelos superficiales a medianamente profundo, con un promedio de 0.30 $\mathrm{m}$; textura ligera a media, $p \mathrm{H}$ de moderado a ligeramente básico (5.98 a 7.99), materia orgánica medio (3.17\%), fósforo disponible medio (8.3 ppm), potasio disponible bajo (Miranda, 1990).

a. Presencia de Trifolium amabile Kunth (Fabaceae)

Para examinar las asociaciones vegetales en las que Trifolium amabile (trébol nativo) está presente, cada sitio se evaluó en cuanto a su composición florística. En cada uno se establecieron en forma aleatoria tres transectos, en los cuales se registró la cobertura basal por señalamiento o "toques" con un anillo censador. Cada transecto fue recorrido utilizando 100 pasos dobles, anotándose en cada paso doble la especie, mantillo, musgo, suelo desnudo, roca y pavimento de erosión (Parker, 1951; citado por Tapia \& Flores, 1984). 
Los datos fueron ingresados en una tabla de doble entrada en donde las columnas representaron a los transectos y las hileras a las especies. Esta tabulación permitió determinar a qué tipo de vegetación se asocia Trifolium amabile así como sus rangos de frecuencia. El análisis estadístico de la información obtenida fue calculado en base a los parámetros estadísticos de frecuencias porcentuales y promedios para las variables evaluadas.

b. Grado de Asociación con Otras Especies Nativas

Para estimar el grado de asociación del trébol nativo con otras especies, en cada sitio y en áreas representativas se establecieron tres transectos de 200 metros cada una, sobre los cuales se muestrearon cada 50 metros con un cuadrante de $0.50 \mathrm{~m}^{2}$ registrándose la presencia y/o la ausencia de Trifolium amabile y las especies acompañantes. Esto permitió acumular un total de 120 observaciones, 12 por sitio para cada zona en estudio. Los datos acumulados fueron ordenados tabularmente en orden alfabético en una matriz de doble entrada. Los nombres científicos de las especies encontradas se ubicaron en las filas y en las columnas los cuadrantes correspondientes a cada sitio.

Los datos de presencia se codificaron con 1 y los de ausencia con 0 (Kent \& Coker, 1992) posteriormente se elaboró una tabla de contingencia 2x2 lo que permitió estimar el grado de asociación por pares de especies encontradas en los sitios evaluados. Esto permitió evaluar cuatro posibilidades, como se muestra a continuación:

\begin{tabular}{cllll}
\multicolumn{3}{c}{ Trifolium amabile } \\
\cline { 2 - 3 } Festuca dolichophylla & + & $\mathrm{a}$ & $\mathrm{c}$ & \\
\cline { 2 - 3 }$(\mathrm{Y})$ & - & $\mathrm{b}$ & $\mathrm{d}$ & $\mathrm{b}+\mathrm{c}$ \\
& & $\mathrm{a}+\mathrm{b}$ & $\mathrm{c}+\mathrm{d}$ & $\mathrm{N}$ \\
\hline
\end{tabular}

En términos de presencia/ausencia; ambas especies se hallan presentes (celda a), T. amabile puede estar presente pero no $F$. dolichophylla (celda b); $F$. dolichophylla puede estar presente pero T. amabile no (celda c); o ninguna de las dos puede estar presente (celda d). Así sucesivamente se procede analizar $T$. amabile con todas las demás especies acompañantes.

El valor de Ji-cuadrado se obtuvo a partir de los datos binomiales de la tabla de contingencia, como una medida de asociación de dos especies (Ec. 1).

$$
\mathrm{X}^{2}=([\mathrm{ad}-\mathrm{bc}]-0.5 \mathrm{~N})^{2} \times \mathrm{N} /(\mathrm{a}+\mathrm{b})(\mathrm{c}+\mathrm{d})(\mathrm{a}+\mathrm{c})(\mathrm{b}+\mathrm{d})
$$$$
\text { (Ec. 1) }
$$

Donde: $\mathrm{a}=$ Suma del valor de las especies $\mathrm{XY}$ cuando están juntas, $\mathrm{b}=$ Suma del valor de la especie $\mathrm{X}$, cuando Y está ausente, $\mathrm{c}=$ Suma del valor de la especie Y cuando X está ausente, $\mathrm{d}=$ Suma del valor de las especies XY cuando están ausentes, $\mathrm{N}$ = Suma total.
La asociación se consideró positiva, cuando la frecuencia observada de las ocurrencias conjuntas de las especies XY en las tablas de contingencia fue mayor a la frecuencia esperada y, en caso contrario la asociación se considera negativa y las especies raramente podrían encontrarse juntas (Ec. 2) (Kent \& Coker, 1992).

Frecuencia Esperada $=(\mathrm{a}+\mathrm{b}) \times(\mathrm{a}+\mathrm{c}) / \mathrm{N}$

(Ec. 2)

Donde: $\mathrm{a}+\mathrm{b}=$ Suma del número de ocurrencias de la especie $\mathrm{X} ; \mathrm{a}+\mathrm{c}=$ Suma del número de ocurrencias de la especie $\mathrm{Y}$; $\mathrm{N}$ = Suma total.

\section{c. Factores Abióticos y Presencia de Trifolium amabile Kunth (Fabaceae)}

En cada punto de muestreo de la vegetación se evaluaron los factores del suelo que podrían influir en la presencia/ausencia de Trifolium amabile como la textura, la profundidad, la temperatura, la humedad, la concentración de nitrógeno total, el fósforo disponible y el $\mathrm{pH}$. Los análisis físico-químicos de las muestras de suelo se realizaron en el Laboratorio de suelos de la UNA-Puno. Para el análisis de los datos se utilizó la prueba de correlación no paramétrica de Spearman (nivel de significancia $\mathrm{p}<0.05$ y $\mathrm{p}<0.20$ ) (Bajsa, 2008).

\section{Resultados y discusión.}

a. Presencia de Trifolium amabile Kunth (Fabaceae)

Trifolium amabile se encuentra presente en todas las asociaciones vegetales estudiadas y su porcentaje de presencia varió de 1.0 a 8.3 \% (Tabla 1). Los mayores porcentajes de $T$. amabile se observaron en aquellos sitios donde las especies Festuca dolichophylla y Muhlenbergia fastigiata eran dominantes.

No hubo diferencias significativas $(\mathrm{p}=0.087)$ en los porcentajes promedio de presencia entre Ilpa (3.7 \%) y Quimsachata (3.4\%). Los valores más elevados de presencia de esta especie fueron $6.3 \%$ y $8.3 \%$ para ambos sitios, respectivamente (Tabla 1). Porcentajes de $5.3 \%$ de presencia de $T$. amabile fueron reportados por Wilcox \& Bryant (1984) en pajonales ubicados en laderas montañosas, de la sierra central del Perú, dominados por Festuca distichovaginata y Stipa brachiphylla. Florez y Malpartida (1987) reportaron para los andes centrales, presencia de Trifolium amabile en porcentajes por debajo de $5 \%$ de la composición florística atribuyéndose estos bajos porcentajes al pastoreo intenso de ovinos y camélidos prevalente en la zona alto andina.

El hecho de que se encuentre presente en los sitios estudiados pero en pequeño porcentaje, menos del 10 $\%$, sugiere que si bien T. amabile posee mecanismos de adaptación a factores abióticos (sequia, heladas, calidad de suelos, fluctuaciones amplias de temperaturas entre el día y la noche) y bióticos 
(presión de herbívoro y competencia de gramíneas nativas) estarían limitando su mayor presencia. A pesar de estos porcentajes bajos, esto no reduce su importancia ecológica por el rol que estaría cumpliendo en la fijación de nitrógeno y su aporte de proteína en la dieta de

los animales, mas aun si se tiene en cuenta que el valor proteico de las gramíneas nativas dominantes en los pajonales alto andinos es bajo y durante la época seca cae muy por debajo de aquellos niveles considerados críticos para el mantenimiento de las bacterias ruminales (Aguirre \& Flores, 2012).

Los mayores porcentajes de T. amabile en la zona de Quimsachata, se encontró en tres asociaciones vegetales dominadas por gramíneas: Muhlembergia fastigiata - Festuca dolichophylla - Stipa ichu/ Festuca dolichophylla - Muhlenbergia fastigiata Stipa obtusa/ y Muhlenbergia fastigiata - Stipa obtusa - Festuca dichoclada (Tabla 1). En la zona de Illpa, los mayores porcentajes de $T$. amabile se encontró en cuatro asociaciones vegetales dominadas por: Muhlenbergia fastigiata - Distichlis humilis Festuca dolichophylla/ Distichlis humilis Muhlenbergia fastigiata - Festuca dolichophylla/ Muhlenbergia fastigiata - Festuca dolichophylla Aristida adcensionis/ y Muhlenbergia fastigiata Festuca dolichophylla - Calamagrostis curvula (Tabla 1).

Una tendencia general es que $T$. amabile estuvo siempre asociada a Festuca dolichophylla (gramínea alta) y Muhlembergia fastigiata (gramínea de porte bajo) en ambas zonas; lo que podría indicar que la presencia de esta especie es favorecida por las condiciones en las que se desarrollan estas dos gramíneas (suelos profundos con buena humedad y drenaje), así como por las modificaciones micro ambientales que estarían propiciando estas gramíneas para la presencia de T. amabile, favoreciendo en su protección contra el frío y vientos intensos. De otro lado el paisaje colinoso, con presencia de quebradas podría estar favoreciendo la mayor presencia de $T$. amabile en la zona de Quimsachata en comparación a un paisaje de planicie que presenta la zona de Illpa; donde la velocidad de los vientos y la magnitud de los cambios de temperatura entre el día y la noche son mas estresantes (Daubenmire, 1996).

Otro factor que podría haber contribuido al mantenimiento de condiciones más favorables para el Trifolium amabile (Tabla 1) es el mantillo, el cual se encontró en mayor proporción en Quimsachata 1.9\% comparada con Illpa $1.2 \%$. El mantillo es un componente primordial en la protección del suelo y de plántulas y podría haber jugado un rol importante en el mantenimiento de mejores condiciones de temperatura y estatus hidrológico para el T. amabile en Quimsachata (European Food Safety Authority, 2010; Porta et al., 1999; Daubenmire, 1996.

os sitios con mayor frecuencia de Trifolium amabile

\begin{tabular}{lllllll} 
Aziruni & Cotosa & Illpa & Collana & Colca & Vilque \\
Fa & Fedo & Mufa & Mufa & Dihu & Mufa & Mufa \\
Mufa & Stob & Dihu & Mufa & Fedo & Fedo \\
& Stob & Fedi & Fedo & Fedo & Arad & Cacu \\
& 94.7 & 79.3 & 93.3 & 92.7 & 91.0 & 89.7 \\
0.7 & 2.7 & 0.3 & 0.7 & 3.0 & 0.7 \\
4.7 & 11.7 & 6.3 & 6.7 & 6.0 & 9.7 \\
$\mathbf{5 . 3}$ & $\mathbf{5 . 3}$ & $\mathbf{5 . 3}$ & $\mathbf{5 . 0}$ & $\mathbf{6 . 0}$ & $\mathbf{6 . 3}$ \\
\hline
\end{tabular}

Mufa=Muhlenbergia fastigiata, Fedo=Festuca dolichophylla, Stob=Stipa obtusa, Stich=Stipa ichu, Fedi=Festuca dichoclada, Dihu=Distichlis humilis, Arad=Aristida adcensionis, $\quad \mathbf{C a c u}=$ Calamagrostis curvula.

b. Grado de asociación con otras Especies Nativas

El análisis del grado de asociación de T. amabile con otras especies, reveló para la zona de Quimsachata, la existencia de siete asociaciones de naturaleza positiva con, gramíneas de porte alto: Festuca dolichophylla y Poa horridula, gramíneas de porte bajo: Muhlenbergia fastigiata, herbáceas: Alchemilla pinnata, Eleocharis albibracteata, Azorella diapensoides, y Geranium sessiliflorum. Se encontraron cinco asociaciones de naturaleza negativa con las gramíneas Calamagrostis amoena, Festuca dichoclada, y Stipa brachiphylla y con las arbustivas Margiricarpus pinnatus y Parastrephia quadrangularis. En tanto, que en la zona de Illpa, se encontró una asociación positiva con Eleocharis albibracteata y otra negativa con Distichlis humilis y Stipa ichu (Tabla 2).

La naturaleza de la asociación positiva que se encontró entre T. amabile con gramíneas altas como Festuca dolichophylla y Poa horridula, corrobora lo indicado, en relación a las comunidades vegetales, donde éstas gramíneas junto con la leguminosa nativa se desarrollan en suelos profundos y húmedos (Tapia \& Flores, 1984) y condiciones ecológicas de ladera baja, planicies con ligera pendiente y, están distribuidas entre los 3800 a 4500 metros de altitud (Tovar, 1988). Adicionalmente, la asociación positiva entre estas especies podría estar siendo favorecida por la diferencia en la morfología y profundidad radicular de estas dos especies, dado que las gramíneas presentes tienen un sistema radicular fibroso y poco profundo en comparación con la raíz pivotante y profunda que posee la leguminosa, lo que estaría permitiendo la exploración de diferentes estratos de suelo para la coexistencia de las gramíneas nativas con T. amabile. 
Ecol. apl. Vol. 12 № 2, pp. 83-89

Tabla 2. Medida y naturaleza de asociatividad entre Trifolium amabile y otras especies en dos zonas de la pradera nativa de Puno.

\begin{tabular}{|c|c|c|}
\hline Zonas/Especies & $\begin{array}{c}\text { Prueba } \\
\text { de Ji- } \\
\text { cuadrada }\end{array}$ & $\begin{array}{c}\text { Naturaleza } \\
\text { de } \\
\text { asociación }\end{array}$ \\
\hline \multicolumn{3}{|l|}{ Quimsachata } \\
\hline Alchemilla pinnata & $10.56^{\mathrm{a}}$ & + \\
\hline Azorella diapensoides & $6.53^{\mathrm{a}}$ & + \\
\hline Calamagrostis amoena & $16.33^{\mathrm{a}}$ & - \\
\hline Eleocharis albibracteata & $3.15^{\mathrm{b}}$ & + \\
\hline Festuca dichoclada & $4.19^{a}$ & - \\
\hline Festuca dolichophylla & $14.25^{\mathrm{a}}$ & + \\
\hline Geranium sessiliflorum & $2.09^{b}$ & + \\
\hline Margiricarpus pinnatus & $2.58^{\mathrm{b}}$ & - \\
\hline Muhlenbergia fastigiata & $7.10^{\mathrm{a}}$ & + \\
\hline Parastrephia quadrangularis & $2.09^{b}$ & - \\
\hline Poa horridula & $2.79^{\mathrm{b}}$ & + \\
\hline Stipa brachiphylla & $2.21^{\mathrm{b}}$ & - \\
\hline \multicolumn{3}{|l|}{ Illpa } \\
\hline Distichlis humilis & $3.53^{b}$ & - \\
\hline Eleocharis albibracteata & $1.94^{\mathrm{b}}$ & + \\
\hline Stipa ichu & $2.26^{\mathrm{b}}$ & - \\
\hline
\end{tabular}

superficiales, que habrían limitado la presencia de $T$. amabile (Tapia \& Flores, 1984).

c. Factores Abióticos y Presencia de Trifolium amabile Kunth (Fabaceae)

El análisis de correlación entre características del suelo y la presencia de $T$. amabile reveló asociación significativa con la profundidad efectiva del suelo, contenido de nitrógeno y fósforo, sin embargo, la temperatura, humedad del suelo y $\mathrm{pH}$ no mostraron asociación significativa con esta leguminosa. Cuando se examinó, como esta relación variaba con el lugar, se encontró que en la zona de Quimsachata la profundidad efectiva fue el factor más relevante para explicar la presencia de T. amabile, pero no en Illpa (Tabla 3).

La alta correlación de Spearman para profundidad del suelo en Quimsachata ( $\mathrm{r}=0.66$; $\mathrm{p}=0.0015)$ y la baja correlación para este factor en la zona de Illpa, estaría sugiriendo que la profundidad es un factor que varía dependiendo de la naturaleza del sitio, para la distribución de T. amabile. La zona de Illpa, está dominada por extensas planicies y suelos uniformes de mediana profundidad, mientras que Quimsachata presenta topografía montañosa y profundidad del suelo no es uniforme, por lo general, es superficial. En estas condiciones T. amabile no está distribuido uniformemente en todos los sitios del pastizal,
Como resultado de que Festuca dolichophylla, Muhlenbergia fastigiata y otras herbáceas como Alchemilla pinnata, Azorella diapensoides, Eleocharis albibracteata y Geranium sessiliflorum forman parte de una asociación vegetal, $T$. amabile estuvo asociada positivamente con este conjunto de especies y negativamente con gramíneas altas Calamagrostis amoena, Festuca dichoclada, Stipa ichu y Stipa brachiphylla, cuya presencia está relacionada a suelos superficiales, pedregosos propios de laderas y cerros con mucha pendiente, condiciones donde éstas prosperan, serían diferentes a las que la leguminosa requiere. Estas áreas durante el invierno soportan sequías fuertes $\mathrm{y}$ temperaturas ambientales bajas; que podrían ser una limitante para la presencia de T. amabile.

Los resultados también revelan una asociación negativa con Distichlis humilis, sugiriendo que la leguminosa nativa no sería tolerante a suelos salinos, característicos de los suelos con pobre drenaje en los que es común observar la presencia de $D$. humilis (Ortuño et al., 2006). También existe asociación negativa con las especies arbustivas Parastrephia quadrangularis y Margiricarpus pinnatus, típicas de laderas y cerros de baja cobertura vegetal y fisiografía con marcada presencia de afloramientos rocosos y
Tabla 3. Coeficiente de correlación de Spearman entre Trifolium amabile y las propiedades físico-químicas del suelo

\begin{tabular}{llll}
\hline Características físicas & Presencia & $\begin{array}{l}\text { Correlación } \\
\text { Spearman }\end{array}$ & p-value \\
\hline Illpa & & & \\
Profundidad efectiva (cm) & $36.88 \pm 2.22$ & 0.23 & 0.3392 \\
Temperatura suelo $\left({ }^{\circ} \mathrm{C}\right)$ & $12.23 \pm 0.64$ & 0.09 & 0.7162 \\
Humedad suelo $(\%)$ & $20.64 \pm 1.79$ & 0.03 & 0.8846 \\
Nitrógeno (\%) & $0.199 \pm 0.02$ & 0.51 & 0.0227 \\
Fósforo (ppm) & $13.72 \pm 1.10$ & 0.28 & 0.2344 \\
pH & $5.83 \pm 0.18$ & -0.1 & 0.6874 \\
Quimsachata & & & \\
Profundidad efectiva (cm) & $35.62 \pm 2.45$ & 0.66 & 0.0015 \\
Temperatura suelo ( $\left.{ }^{\circ} \mathrm{C}\right)$ & $12.87 \pm 0.62$ & 0.14 & 0.5595 \\
Humedad suelo $(\%)$ & $24.98 \pm 1.19$ & 0.17 & 0.4665 \\
Nitrógeno (\%) & $0.293 \pm 0.02$ & 0.57 & 0.0093 \\
Fósforo (ppm) & $11.12 \pm 1.21$ & 0.48 & 0.0332 \\
pH & $5.44 \pm 0.12$ & -0.18 & 0.4407 \\
\hline
\end{tabular}

presentándose con mayor frecuencia en laderas bajas en suelo con una profundidad efectiva mayor a 0.30 $\mathrm{m}$.

El análisis de los factores químicos indica la existencia de una correlación significativa $(\mathrm{p}<0.05)$ entre el nivel de nitrógeno y la presencia de $T$. amabile, tanto en Quimsachata como en Illpa. La mayor concentración de nitrógeno asociada a la presencia de layo, sugiere que esta leguminosa nativa estaría modificando favorablemente las condiciones de suelo en los que está presente a través de la fijación de 
nitrógeno. En la zona de Quimsachata se observó, que la presencia de $T$. amabile estuvo positivamente correlacionada $(r=0.48 ; p=0.03)$ con la concentración de fósforo disponible en el suelo (Tabla 3). Estos resultados estarían confirmando los mayores requerimientos de este elemento por parte de las leguminosas con respecto a las gramíneas.

En Illpa la correlación no fue significativa probablemente porque se trata de una zona uniforme en contenido de fósforo disponible a nivel medio.

La posible fijación simbiótica de nitrógeno por T.amabile, estaría favorecida por el nivel de fósforo medio, encontrados en ambas zonas. Estudios revelan que las leguminosas nativas fijan nitrógeno a niveles mas bajos que leguminosas mejoradas, sin embargo las razones de esta menor capacidad de fijación deberían ser dilucidadas. Esta desventaja sin embargo, estaría siendo compensada por su mejor adaptación a condiciones donde leguminosas mejoradas no pueden establecerse y persistir con éxito, debido a su marcada sensibilidad a la deficiencia de fósforo, acidez y salinidad, factores que en conjunto como sugiere la literatura afectan la nodulación y la actividad de la nitrogenasa (Serraj \& Adu-Gyamfi, 2004; Sheaffer \& Evers, 2007; Leep, 2007).

\section{Conclusiones.}

Trifolium amabile se encontró en la mayoría de los sitios estudiados, pero en porcentajes relativamente bajos, menos del 8\% de frecuencia; estos porcentajes, sin embargo, no deberían interpretarse como que esta especie no sería ecológicamente importante. El contenido de proteína de las gramíneas dominantes en los pajonales es bajo, al igual que el nivel de nitrógeno en el suelo; por lo que, el eventual aporte de nitrógeno de esta leguminosa nativa al ciclo de nutrientes podría estar jugando un rol en el mantenimiento del estatus nutricional del ecosistema a través de la fijación de nitrógeno atmosférico, aspecto que está aún por determinar.

En relación a la presencia de Trifolium amabile en asociación con otras especies de la comunidad vegetal, los resultados revelaron que esta leguminosa nativa está asociada positivamente con gramíneas de porte alto como Festuca dolichophylla y de porte bajo como Muhlenbergia fastigiata, dos especies forrajeras importantes en la alimentación del ganado, y otras herbáceas que crecen en suelos de mediana profundidad y buen drenaje. Pero está asociada negativamente con gramíneas altas y arbustivas que crecen en laderas con afloramientos rocosos y suelos superficiales.

Un análisis de los factores abióticos prevalentes en los sitios donde se encontró Trifolium amabile revelaron que la presencia de esta especie está asociada significativamente con la profundidad efectiva del suelo, contenido de nitrógeno y fósforo, lo cual estaría indicando que esta especie requiere suelos profundos, aporta nitrógeno al micrositio donde se encuentra y que el fósforo podría actuar como un factor limitante para mayor distribución y frecuencia en el altiplano donde en la mayoría de suelos el nitrógeno y el fósforo son deficientes.

\section{Literatura citada.}

Aguirre L. \& Flores E. 2012. Ecología de leguminosas nativas. Comunicación personal. EPG. Doctorado en Ciencia Animal. Universidad Nacional Agraria La Molina. Lima-Perú.

Alemán F. 1996. Layu-layo. En: Meneses, R., Waaijenberg, H. y Piérola, L. (eds). Leguminosas en la agricultura boliviana. Proyecto Rhizobiología Bolivia Cochabamba, Bolivia. 347-350 p.

Bajsa N. 2008. Efecto del pastoreo bovino sobre la comunidad bacteriana en un suelo de pradera natural. Tesis Maestría en Ciencias Ambientales. Universidad de la República. Montevideo. Uruguay. 77 p.

Cajal J.L., Garcia J. \& Tecchi R. 1998. Bases para la Conservación y manejo de la puna y cordillera frontal de Argentina. FUCEMA. Uruguay. 335 p.

Chaquilla O. 1969. Leguminosas del altiplano peruano. Tesis Ing. Agron. UNTA-Puno.

Daubenmire R.F. 1996. Ecología Vegetal. Tratado de Autoecología de Plantas. México. 496 p.

De La Cruz H. 2007. Uso tradicional (Medicinal y biocida) de las especies vegetales silvestres de la Cuenca del Río Chillon, Canta-Lima. UNA-La Molina. Tesis Ph.D. Lima-Perú.

European Food Safety Authority. 2010. Scientific opinión on the importance of the soil litter layer in agricultural areas. EFSA Journal. Parma, Italy. 8(6): 1625.

FAO. 2005. Grassland of the World. Edited by Suttie, J. M.; Reynolds, S.G., and Batello, C. Rome, Italy.

Flores E. 1991. Manejo y utilización de pastizales. En: Avances y perspectivas del conocimiento de los camélidos sudamericanos. FAO. Santiago, Chile. 429 p.

Florez A. 2005. Manual de Pastos y Forrajes Altoandinas. OIKOS. Lima-Perú. 53 p.

\& Malpartida E. 1987. Manejo de praderas nativas y pasturas en la región alto andina del Perú. Banco Agrario. Fondo del libro. Tomo I. Lima-Perú. $335 \mathrm{p}$.

Malpartida E. \& San Martín F. 1992. Manual de forrajes para zonas áridas y semiáridas andinas. Red de Rumiante, Menores. RERUMEN. Lima-Perú. 281 p.

Hyland CH., Ketterings Q., Dewing D., Stockin K., Czmmek K., Albrecht G. \& Geohring L. 2005. Phosphorus basics-The phosphorus cycle. Fact Sheet 12. Cornell University. 12 p.

Kent M. \& Coker P. 1992. Vegetation description \& Analysis: a practical approach. Belhaven Press. London. R.U. 355 p.

Leep R.H. 2007. Fertilization. Michigan State University, East Lansing. 759: 355-377 p. In: Forages The Science of Grassland Agriculture. Under the editorial authorship of Barnes, R. F.; Nelson, C. J.; Moore, K. J. and Collins, M. $6^{\mathrm{TH}}$ Edition. Volume II. Iowa State University. USA.

Miranda F. 1990. Evaluación edafo-agrostológico de praderas naturales del Centro Experimental Quimsachata-Puno. Tesis Ing. Agrón. Facultad Ciencias Agrarias. UNA. Puno. 
Ecol. apl. Vol. 12 № 2, pp. 83-89

Oficina Nacional de Evaluación de Recursos Naturales. ONERN. 1965. Inventario y evaluación de recursos naturales del departamento de Puno. CORPUNO. PunoPerú.

Pasquín A.I. Grosso L.B. Mangeaud A.P. \& Depetris P.J. 2002. Geoquímica de ríos de montaña en las Sierras Pampeanas. I. Vertientes y arroyos de batolito de Achala, provincia de Córdova, Argentina. Universidad Nacional de Córdova. Argentina. 4: 437-444.

Ortuño T. Beck S. \& Sarmiento L. 2006. Dinámica Sucesional de la vegetación en un sistema agrícola con descanso largo en el Altiplano Central Boliviano. Vol. 41. La Paz, Bolivia. 40-70 pp.

Porta J. Lopez-Acevedo M. \& Roquero C. 1999. Edafología para la agricultura y el medio ambiente. 2da. Edición. Edic. Mundi-Prensa. España. 849 p: 155-193.
Serraj R. \& Adu-Gyamfi J. 2004. Role of symbiotic Nitrogen fixation in the improvement of legume productivity under stressed environments. West African Journal of Applied Ecology. Vol. 6. 95-109 p.

Sheaffer C. C. \& Evers G. W. 2007. Cool-Season Legumes for Humid Areas, pp. 179-190. In: R.F. Barnes, et al. (eds.), Forage The Science of Grassland Agricultura. Volume II. 6Th Edition. Iowa State. USA.

Tapia M.E. \& Flores J.A. 1984. Pastoreo y Pastizales de los Andes del Sur del Perú. INIPA. Lima-Perú.

Tovar O. 1988. Manual de identificación de pastos naturales de los andes del sur peruano. (Gramíneas). Proyecto Alpacas/COTEZU/IC. Lima-Perú.

Wilcox B. \& Bryant F. 1984. Asociaciones vegetales en las praderas naturales de la sierra central del Perú. Rumiantes Menores -Perú. Lima-Perú.

\footnotetext{
${ }^{1}$ Estación Experimental Agraria Illpa-INIA, Rinconada de Salcedo s/n. Puno-Perú. gargote@inia.gob.pe.

${ }^{2}$ Laboratorio de Ecología y Utilización de Pastizales. Facultad de Zootecnia. Universidad Nacional Agraria La Molina. Avenida La Molina 1 980. Lima-Perú. laterra@lamolina.edu.pe, efm@lamolina.edu.pe.

${ }^{3}$ Laboratorio de Ecología y Utilización de Pastizales. Facultad de Zootecnia. Universidad Nacional Agraria La Molina. Avenida La Molina 1 980. Lima-Perú. laterra@lamolina.edu.pe, efm@lamolina.edu.pe.
} 